\title{
Magnetic field dependence of the low-energy spectrum of a two-electron quantum dot
}

\author{
C. E. Creffield, ${ }^{1,3}$ J. H. Jefferson, ${ }^{2}$ Sarben Sarkar, ${ }^{3}$ and D. L. J. Tipton ${ }^{2,3}$ \\ ${ }^{1}$ Instituto de Ciencia de Materiales (CSIC), Cantoblanco, E-28049, Madrid, Spain \\ ${ }^{2}$ Defence Evaluation and Research Agency, Electronics Sector, St. Andrews Road, Malvern, Worcs. WR14 3PS, United Kingdom \\ ${ }^{3}$ Department of Physics, King's College London, Strand, London, WC2R 2LS, United Kingdom
}

(Received 21 January 2000)

\begin{abstract}
The low-energy eigenstates of two interacting electrons in a square quantum dot in a magnetic field are determined by numerical diagonalization. In the strong correlation regime, the low-energy eigenstates show Aharonov-Bohm-type oscillations, which decrease in amplitude as the field increases. These oscillations, including the decrease in amplitude, may be reproduced to good accuracy by an extended Hubbard model in a basis of localized one-electron Hartree states. The hopping matrix element $t$ comprises the usual kinetic energy term plus a term derived from the Coulomb interaction. The latter is essential to get good agreement with exact results. The phase of $t$ gives rise to the usual Peierls factor, related to the flux through a square defined by the peaks of the Hartree wave functions. The magnitude of $t$ decreases slowly with magnetic field as the Hartree functions become more localized, giving rise to the decreasing amplitude of the Aharonov-Bohm oscillations.
\end{abstract}

\section{INTRODUCTION}

Advances in the fabrication of semiconductor nanostructures have made it possible to construct devices, termed "quantum dots", in which a small number of electrons can be confined to regions of the order of the Fermi wavelength. Due to this confinement the electronic spectrum of a quantum dot is composed of discrete levels, which have been studied in detail by conductance and spectroscopic measurements. Interactions between electrons have been shown to be of major importance in determining the electronic properties of these systems, and the effects of the strong correlations between particles has attracted intense experimental and theoretical attention. A particular motivation for studying the properties of few electron quantum dots is their relevance to the rapidly developing field of quantum computing, ${ }^{1}$ as the entangled states of the electrons confined in a quantum dot can give a physical realization of a quantum bit or "qubit." A convenient probe to study quantum dots is the application of a magnetic field that has revealed numerous dramatic and novel quantum effects, such as parity oscillations of the ground state $^{2}$ and the phenomenon of magic numbers. ${ }^{3} \mathrm{~A}$ variety of techniques have been developed to treat these systems, including the numerical quantum Monte Carlo method (QMC), ${ }^{4,5}$ Hartree-Fock diagonalization, ${ }^{6}$ and the direct diagonalization of the many-body Hamiltonian. ${ }^{3}$ The majority of these treatments, however, have concentrated on circularly symmetric dots with a parabolic confining potential. This is indeed a reasonable approximation to the potential found experimentally in large dots produced by "soft" confinement, and it is appealing from the theoretical point of view because the rotational symmetry of the potential renders the singleparticle problem completely integrable, ${ }^{7,8}$ giving a natural basis to describe the many-particle situation. In real devices, however, one must expect deviations from perfect symmetry, which can have profound effects on their spectrum. ${ }^{9}$ For example, "hard confined"' quantum dots produced by StranskiKrastanov growth has abrupt heterojunction interfaces in all directions, and the parabolic approximation is unrealistic since the potential is essentially flat within the dot, rising sharply at the dot boundary. A square well bounded by infinite potential barriers provides a simple, though idealized, model of this form of confinement. In contrast to rotationally symmetric potentials, angular momentum is not a good quantum number for the square-well system except in the limit of very high fields, and accordingly even the single-particle problem is nonintegrable. Another consequence of the square symmetry is that this system can exhibit many-body effects that are not readily observed in parabolically confined dots, ${ }^{10}$ as the abrupt boundaries mean that the spatial coordinates cannot be split into center of mass and relative coordinates, and hence Kohn's theorem is not applicable.

In this paper we consider the case of two electrons confined to a square-well quantum dot in the presence of a magnetic field. In a square-well potential the kinetic energy scales like $1 / L^{2}$ and the interaction energy like $1 / L$, where $L$ is the side length of the well. The competition between these terms determines the nature of the electron system. For small $L$ the Coulomb energy is insignificant in comparison to the single-particle kinetic energies and the electrons behave like uncorrelated independent particles. As $L$ increases the Coulomb term becomes increasingly dominant and the manyparticle states become correlated and cannot be described in terms of an independent-electron picture. In this limit the electrons will form a quasicrystalline state to minimize their electrostatic energy, termed a "Wigner molecule" in analogy with the formation of a Wigner crystal in an infinite system. ${ }^{11}$ This observation is the key to a method developed to treat the strongly correlated situation by mapping the lowenergy spectrum of the system to an effective lattice model of the Hubbard type, ${ }^{12}$ where the lattice points are identified with the peaks in the charge density of the Wigner molecule state. In the absence of a magnetic field this technique has been successfully used to treat one-dimensional quantum dots containing up to six electrons, ${ }^{12}$ and also for twodimensional polygonal dots containing two electrons. ${ }^{13}$ If 
this mapping to a lattice model indeed captures the basic physics of the system, we should expect it to also reproduce the dominant effects of an applied magnetic field by the inclusion of a Peierls factor ${ }^{14}$ in the intersite hopping terms. We test this expectation here by comparing the predictions of the lattice model modified in this way with results obtained by the explicit diagonalization of two-electron quantum dots pierced by a variety of different magnetic flux distributions. We find that in all cases the Peierls substitution gives the correct qualitative behavior of the energy levels, and becomes precise in the limit of an Aharonov-Bohm flux line. Quantitative accuracy for physically realizable magnetic fields may be obtained by minor renormalizations of the parameters of the effective lattice model, arising from the increased localization of the electron wave functions produced by a physical magnetic field. We further justify this picture by explicitly constructing localized Hartree basis functions. The Hamiltonian is then diagonalized using the lowest four Hartree basis functions, corresponding to an electron localized near one of the four corners of the square. This gives good agreement with the exact results. Furthermore, retaining only nearest-neighbor hopping and diagonal Coulomb repulsion terms also gives good quantitative agreement with the exact result, provided that the hopping term includes an important contribution from the Coulomb interaction. This gives explicit justification of the simple extended Hubbard model and correctly reproduces the detailed dependence of the energy levels on the applied field. Finally we discuss the relevance of these results to the phenomena predicted to occur in quantum dot arrays, ${ }^{15}$ which can also be described by a mapping to a lattice model of Hubbard type.

\section{LOW-ENERGY EIGENSTATES}

We consider a square quantum dot, described by the Hamiltonian

$$
H=\frac{1}{2 m^{*}} \sum_{i=1}^{2}\left[\left(-i \hbar \nabla_{i}+e \mathbf{A}_{i}\right)^{2}+V\left(\mathbf{r}_{i}\right)\right]+\frac{e^{2}}{4 \pi \epsilon_{0} \epsilon_{r}} \frac{1}{\left|\mathbf{r}_{1}-\mathbf{r}_{2}\right|},
$$

where $V(\mathbf{r})$ is the confinement potential and $\mathbf{A}$ is the vector potential of the applied magnetic field $\mathbf{B}$. We assume that the electrons are confined by infinite walls, and that they can be described by the effective-mass approximation. The two simplest forms of applied magnetic field are a uniform field, $B_{z}$, perpendicular to the plane of the dot and, at the other extreme, an Aharonov-Bohm flux line in which the magnetic field is zero throughout the dot except at a single point, but phase interference effects arise from the particles' interactions with the vector potential. We may interpolate between these cases by using a flux tube of radius $a$, inside which the magnetic field is uniform and outside of which the field is zero. This is equivalent to the field produced by an infinitely long solenoid of radius $a$. In the symmetric gauge it can easily be shown that the vector potential corresponding to this field is given by

$$
\mathbf{A}=\left(A_{r}, A_{\theta}\right)= \begin{cases}\left(0, \frac{B r}{2}\right) & \text { for } r \leqslant a \\ \left(0, \frac{B a^{2}}{2 r}\right) & r>a .\end{cases}
$$

However, to simplify the numerical investigation it was decided to use an analytic form for the vector potential:

$$
A_{\theta}=\frac{B a^{2}}{2} \frac{r}{r^{2}+a^{2}},
$$

which has the same behavior as Eq. (2) at small and large values of $r$ but does not have a cusp at $r=a$ and is therefore more amenable to a numerical treatment. Aligning the flux tube with the center of the dot allows us to easily compare the results obtained as the magnetic field is changed from the uniform case $(a \rightarrow \infty)$ toward the limit of an AharonovBohm flux line $(a=0)$ by altering the value of $a$.

To find the eigenvalues of the Hamiltonian (1) we chose to use a basis of states $\left\{\psi_{n}(x) \psi_{m}(y)\right\}$ for each electron, where

$$
\psi_{n}(x)=\sqrt{\frac{2}{L}} \sin \left(\frac{n \pi x}{L}\right), \quad 0 \leqslant x \leqslant L
$$

and $L$ is the side length of the square dot, which are the eigenstates of the noninteracting system in the absence of a magnetic field. For the specific case of a uniform magnetic field the matrix elements of all the single-electron terms in the Hamiltonian (1) can be calculated analytically in this basis. For the other field configurations, however, it was necessary to calculate these matrix elements numerically, and a NAG routine was used to evaluate the two-dimensional integrals. The matrix elements of the Coulomb interaction were also obtained numerically. Up to eight basis functions per direction were used for each particle to obtain the matrix form of the Hamiltonian, which was then block-diagonalized into singlet and triplet subspaces and treated by a standard eigenvalue routine. In all cases the dot material was taken to be GaAs, with an effective mass $m^{*}=0.067 m_{e}$ and a relative permittivity $\epsilon_{r}=10.9$, giving an effective Bohr radius of $a_{B}$ $=8.8 \mathrm{~nm}$.

We first consider the case of noninteracting electrons in the presence of a uniform magnetic field in order to understand the single-particle effects of the magnetic field, before introducing the additional complication of strong correlations. We show in Fig. 1 the dependence of the energies of the low-lying eigenstates on the strength of the applied field $B_{z}$ for the two noninteracting electrons. At low field strengths the spectrum is extremely complex, with some levels showing a linear dependence on the field and others a quadratic dependence. Many level crossings occur, and also the phenomenon of "avoided crossings" or level repulsion can be clearly seen. This is a typical signature of the presence of quantum chaos and arises here because of the nonintegrability of the single-particle problem in a square boundary. Such avoided crossings are not seen, for example, when the confining boundary is rotationally symmetric. Avoided crossings have also been seen in experiments, such as in Ref. 16, in which electron micrographs of the quantum dots clearly show deviation from circular symmetry. At high 


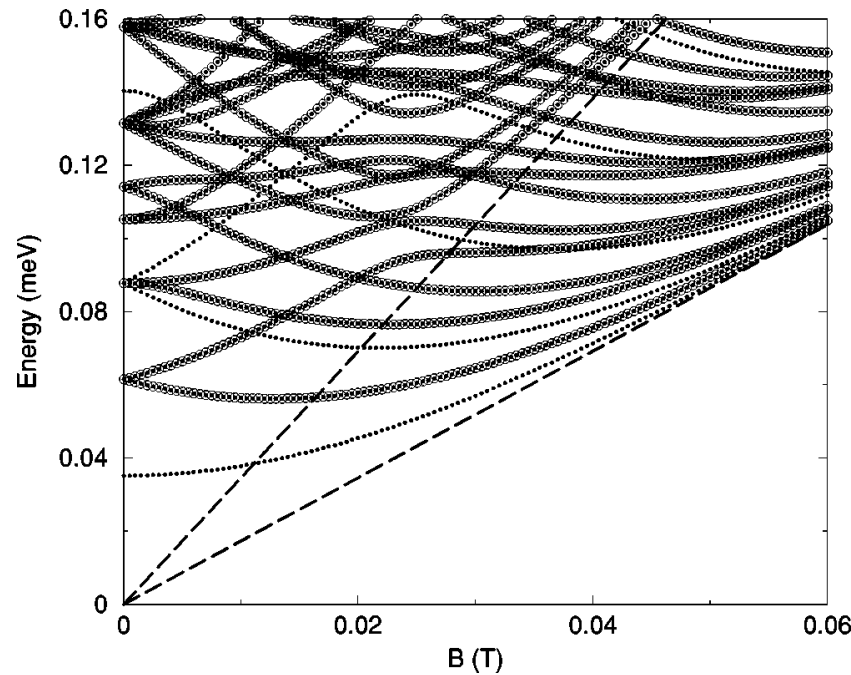

FIG. 1. Energy levels of two noninteracting electrons in a $800 \mathrm{~nm}$ square-well quantum dot. Dots and open circles indicate singlet and triplet levels, respectively. Each triplet level is degenerate with a singlet level. Dashed lines show the lowest two Landau levels.

magnetic fields the spectrum simplifies considerably, and it can be seen that the two-electron energy levels start to condense into highly degenerate Landau levels with a linear dependence on the field, $E_{n}=(n+1) \hbar \omega_{c}$, where $\omega_{c}=e B / m^{*}$ is the cyclotron frequency. It should be noted that the ground state experiences no level crossings and evolves smoothly into the lowest Landau level, remaining a spin singlet for all values of magnetic field.

We now consider the effect of turning on the Coulomb interaction. As was stated earlier, the physical size $L$ of the dot determines the relative importance of the kinetic and Coulomb energies of the system, and when the mean electron separation exceeds a critical value $r_{c}$ the electron charge density becomes localized in space, forming a Wigner molecule. It was found in Ref. 13 that for a two-electron polygonal dot $r_{c} \approx 10 a_{B}$. We show in Fig. 2 the ground-state charge densities in zero magnetic field for two extreme cases $L$ $\ll r_{c}$ and $L \gg r_{c}$ to show how the structure of the ground state alters as $r_{c}$ is exceeded.

In Fig. 3 we show the evolution of the lowest-energy levels as a function of the field strength of a uniform mag-
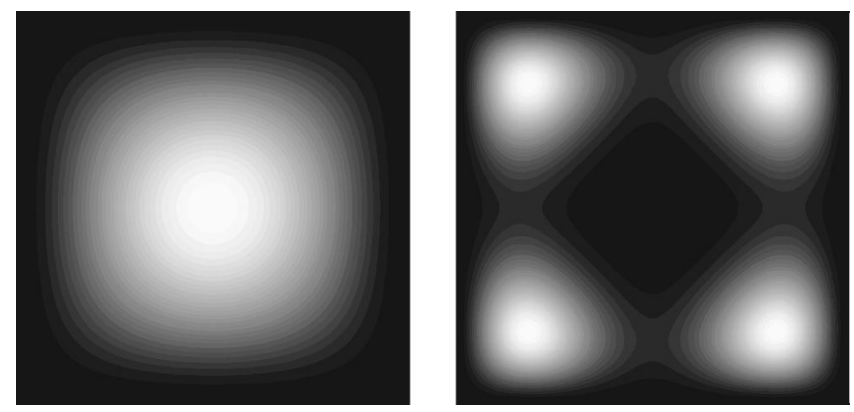

FIG. 2. Ground-state charge-density distributions obtained by exact diagonalzation for (a) $L=10 \mathrm{~nm}$, (b) $L=1600 \mathrm{~nm}$, showing the transition from a weakly interacting case to a sharply peaked Wigner crystal. These peaks define the lattice points that are used in the construction effective charge-spin models (see Sec. III).

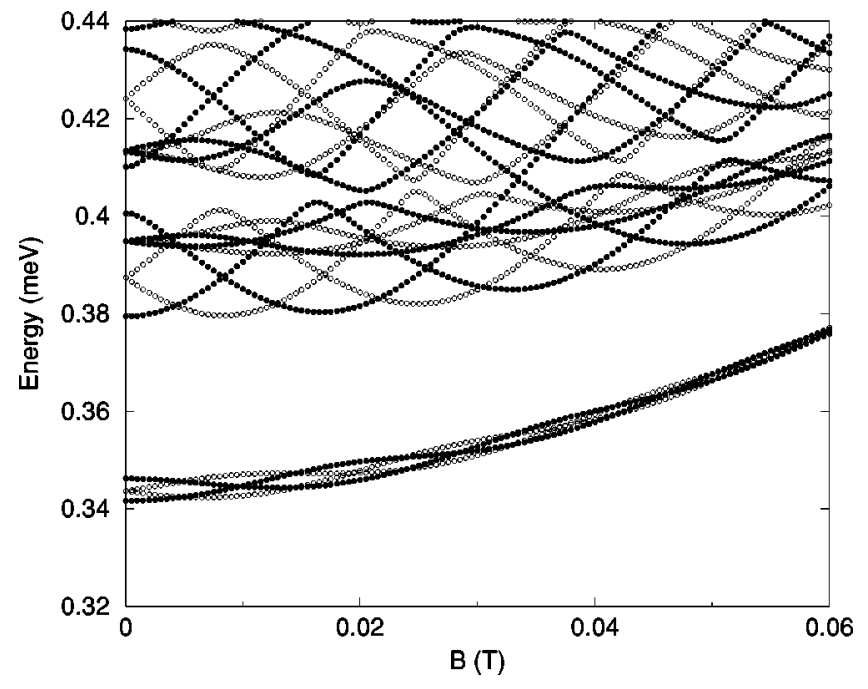

FIG. 3. Energy levels of a two-electron square-well quantum dot in a uniform magnetic field. Filled and open circles indicate singlet and triplet levels, respectively.

netic field. The dot size was taken to be $L=800 \mathrm{~nm}$, which is well within the Wigner molecule regime. We see that there is a low-lying multiplet of two singlet and two triplet states that are relatively well separated from higher-lying states and oscillate with field, with an overall energy increase. To show these oscillations more clearly we subtract the average energy of the multiplet, $E_{0}(B)$, from the eigenenergies, which has an approximately quadratic dependence on applied field (see also Sec. IV). The fine details of the oscillations in energy are then seen clearly, as shown in Fig. 4. In particular, we see that the oscillations have a definite frequency and a gradually decaying envelope. It follows that the ground-state energy oscillates between singlet and triplet with increasing field. We note that the Zeeman term has been neglected in the initial Hamiltonian (1). This would, of course, produce small splittings between the constituent states of each triplet, but otherwise does not change the results in an essential way.

The rest of the paper will focus mainly on this low-lying

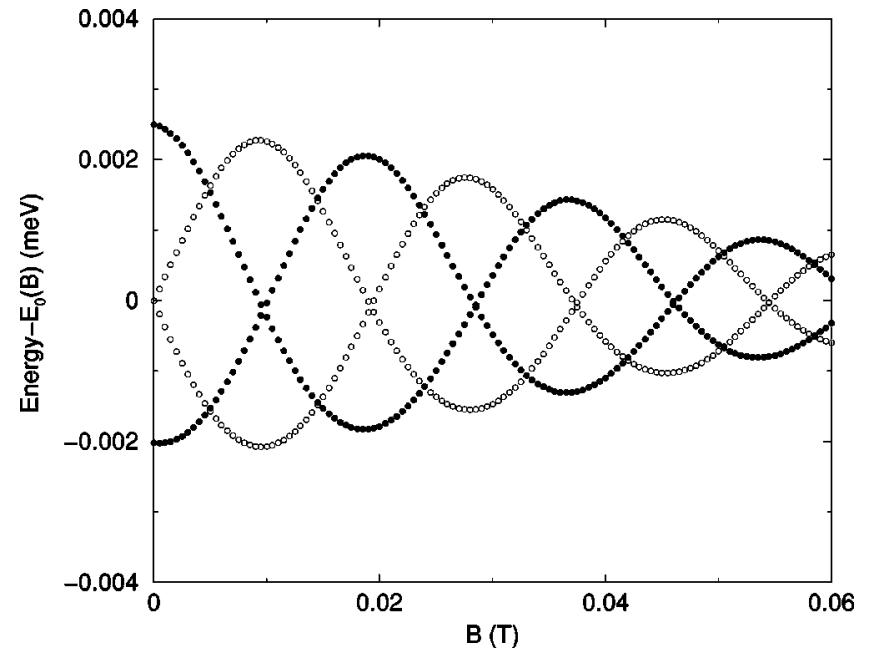

FIG. 4. Dependence of the lowest-lying energy levels on magnetic field, with the average increase $\left[E_{0}(B)\right]$ with field subtracted. Filled and open circles indicate singlet and triplet levels, respectively. 
manifold of states and in the next section we show how it may be described in terms of effective charge-spin models, which in turn explains why the manifold is isolated from higher-lying states, the nature of the oscillations with applied field, and how they may be interpreted in terms of quasi-onedimensional persistent currents.

\section{EFFECTIVE CHARGE-SPIN MODELS AND PERSISTENT CURRENTS}

The existence of the strongly correlated Wigner molecule state is the criterion for the validity of mapping the low-lying states of the quantum dot to an effective lattice model, where the lattice sites are given by the location of the peaks of the Wigner molecule state shown in Fig. 2. In Ref. 12 it was conjectured that the appropriate effective lattice model to describe the low-energy manifold of a system of strongly interacting electrons in a quantum dot is an extended singleband Hubbard model. If, for this case of two electrons, we neglect direct exchange and set the Hubbard $U$ energy to infinity (equivalent to forbidding double occupation of "lattice" sites and neglecting superexchange), then the extended Hubbard model takes on the particularly simple form:

$$
H^{t V}=E_{0}+\mathcal{P}\left[\sum_{\langle i, j\rangle \sigma}\left(t c_{i \sigma}^{\dagger} c_{j \sigma}+\text { H.c. }\right)+V n_{i} n_{j}\right] \mathcal{P} .
$$

Here $\mathcal{P}$ is a projection operator that eliminates doubly occupied lattice sites, $t$ is a nearest-neighbor hopping term, and $V$ is the difference in Coulomb energy between states when the two electrons occupy neighboring sites and when they are diagonally opposite each other.

The energy $E_{0}=2 \varepsilon_{0}+V_{0}$ is the ground-state energy in the "atomic limit", where $\varepsilon_{0}$ is the on-site energy and $V_{0}$ is the Coulomb energy between the two electrons on diagonally opposite sites. The energy parameters $E_{0}, V$, and $t$ depend on the magnitude of the magnetic field, and $t$ in general is complex. One can see on inspection that the spectrum of the Hamiltonian (5) must consist of a low-lying manifold of two singlets and two triplets, corresponding to the electrons being on diagonally opposite sites, and a higher-lying manifold consisting of four singlets and four triplets, corresponding to the two electrons being on neighboring sites. Furthermore, in the strong correlation regime for which $V \gg t$, the lower manifold should have energy $\sim E_{0}$ with the higher-lying manifold at energy $\sim E_{0}+V$. This is indeed the case as can be seen from the exact analytic solutions that take the form

$$
\begin{gathered}
E_{\mathrm{s}}-E_{0}=\frac{1}{2}\left[V \pm \sqrt{V^{2}+16|t|^{2}(1-\cos 2 \phi)}\right], \\
\frac{1}{2}\left[V \pm \sqrt{V^{2}+16|t|^{2}(1+\cos 2 \phi)}\right], \quad V, \quad V
\end{gathered}
$$

for the singlets and

$$
\begin{gathered}
E_{\mathrm{t}}-E_{0}=\frac{1}{2}\left[V \pm \sqrt{V^{2}+16|t|^{2}(1+\sin 2 \phi)}\right], \\
\frac{1}{2}\left[V \pm \sqrt{V^{2}+16|t|^{2}(1-\sin 2 \phi)}\right], \quad V, \quad V
\end{gathered}
$$

for the triplets, where we have set $t=|t| e^{i \phi}$. These solutions are obtained using total $S_{z}$ and fourfold symmetry, which reduces the Hamiltonian matrix to block diagonal form with no more than $2 \times 2$ submatrices. Since $|t| \ll V$, we may expand the square root in Eqs. (6) and (7). To second order this gives for the lowest manifold of states:

$$
\begin{aligned}
& E_{\mathrm{s}}=\widetilde{E}_{0} \pm 2 \Delta \cos 2 \phi, \\
& E_{t}=\widetilde{E}_{0} \pm 2 \Delta \sin 2 \phi,
\end{aligned}
$$

where $\Delta=2|t|^{2} / V$ and $\widetilde{E}_{0}=E_{0}-2 \Delta$. We can, in fact, derive this result in a different way, which emphasizes the nature of the low-lying eigenstates. The excited states corresponding to the two electrons on neighboring sites are eliminated by degenerate perturbation theory. To second order, the $t \mathrm{~V}$-Hamiltonian is then transformed into the effective Hamiltonian: ${ }^{12,13}$

$$
H_{\mathrm{eff}}=\widetilde{E}_{0}+\left(\Delta e^{i 2 \phi} R_{\pi / 2}+\text { H.c. }\right),
$$

where $R_{\pi / 2}$ rotates the two electrons at opposite corners of the square on a diagonal by $\pi / 2$. Diagonalization of $H_{\mathrm{eff}}$ yields directly the singlet and triplet energies (8). In this effective model the pair of electrons thus tunnel between the base states with an amplitude modulated by a Peierls factor, $e^{i 2 \phi}$, with twice the phase angle of the underlying extended Hubbard model, since it involves two electron hops. Although $H_{\text {eff }}$ reproduces the low-energy multiplet of Eq. (5) to good accuracy, it is instructive to calculate the next (fourthorder) correction. This renormalizes $\widetilde{E}_{0}$ and $\Delta$, and also introduces a Heisenberg spin-exchange term:

$$
J\left[\mathbf{s}_{1} \cdot \mathbf{s}_{3}+\mathbf{s}_{2} \cdot \mathbf{s}_{4}\right]
$$

where $J=-\left(16|t|^{4} / V^{3}\right) \cos 4 \phi \quad$ (ferromagnetic). Although this is a small correction, it is not negligible and accounts, for example, for the small asymmetry of the singlet-triplet splittings at $B=0$. The fourth-order effective Hamiltonian has, in fact, the most general form, since higher orders cannot introduce qualitatively different terms. Thus we see that the low-energy manifold can be generally described by a charge-spin model in which the electrons rotate rigidly and undergo Heisenberg exchange. This general effective Hamiltonian [Eqs. (9) and (10)] thus provides a complete and accurate representation of the low-energy spin multiplet and a physical picture of the nature of the states. It is emphasized, however, that such an accurate representation is only valid for the lowest multiplet and that higher-lying states resulting from the solution of the single-band Hubbard model [Eqs. (5)-(7)] are not only less accurate but also incomplete. (There exist further excited states of energy $\sim V$ relative to the ground manifold that are not contained within the approximation of a single-band Hubbard model.)

It is easily verified that Eq. (9) has the correct form to describe the pattern of oscillations seen in Fig. 4 with the quantity $\phi$ being the phase acquired by an electron hopping between two adjacent sites of the lattice on which the effective $t-V$ model is defined. The total phase angle acquired when an electron hops once around the square is $4 \phi$ $=2 \pi \Phi / \Phi_{0}$, where $\Phi_{0}=h / e$ is the fundamental flux quantum and $\Phi=B r_{L}^{2}$ is the total enclosed flux for an effective 


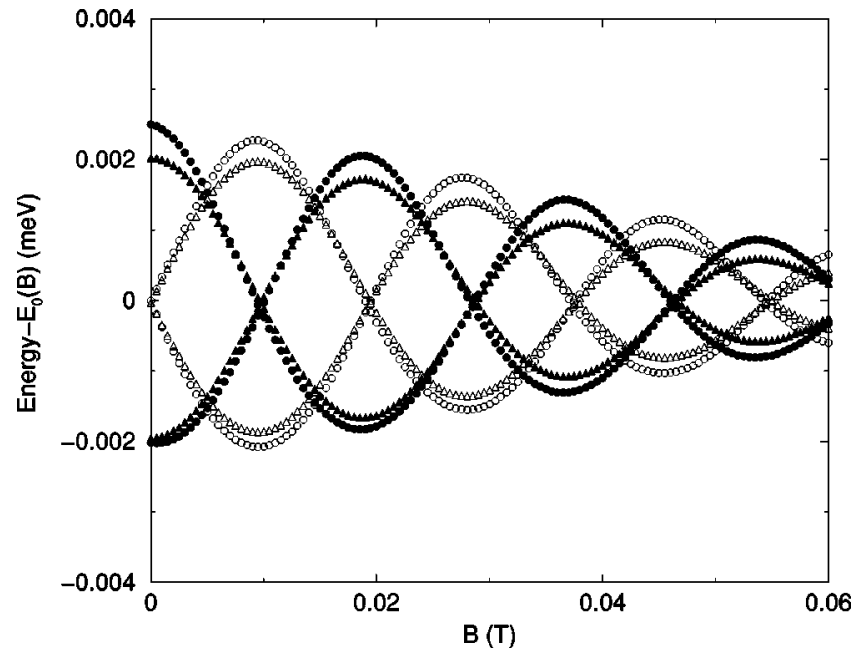

FIG. 5. Comparison of exact calculation (circles) and diagonalization within the space of one Hartree state per site (triangles). Filled and open symbols indicate singlets and triplets, respectively. The overall increase in energy with field $\left[E_{0}(B)\right]$ has been removed.

lattice parameter $r_{L}$. By measuring the distance between the peaks in the ground-state charge distribution we obtain a value of $r_{L}=495 \mathrm{~nm}$ for an $800 \mathrm{~nm}$ dot. Thus a magnetic field of $0.0095 \mathrm{~T}$ corresponds to a magnetic flux of $1.11 \Phi_{0} / 2$, which agrees well with the value of $\Phi_{0} / 2$ that Eqs. (8) predict for the form of the spectrum to become inverted. This close agreement, obtained with no adjustable parameters, fully endorses interpreting the results at nonzero magnetic field within the framework of the lattice model in which the hopping terms are modified by phase factors. An alternative interpretation of this behavior is that the two electrons are hopping around the four sites of the square, giving rise to a persistent current $I=-\partial E / \partial \Phi$. This is periodic in the enclosed flux but with half the period of two noninteracting electrons on a ring, due to the correlated positions of the rotating electrons, described by Eq. (8) It is interesting to note that the inverted spectrum that occurs after a quarter cycle is the result that would be obtained for a bosonic $t-V$

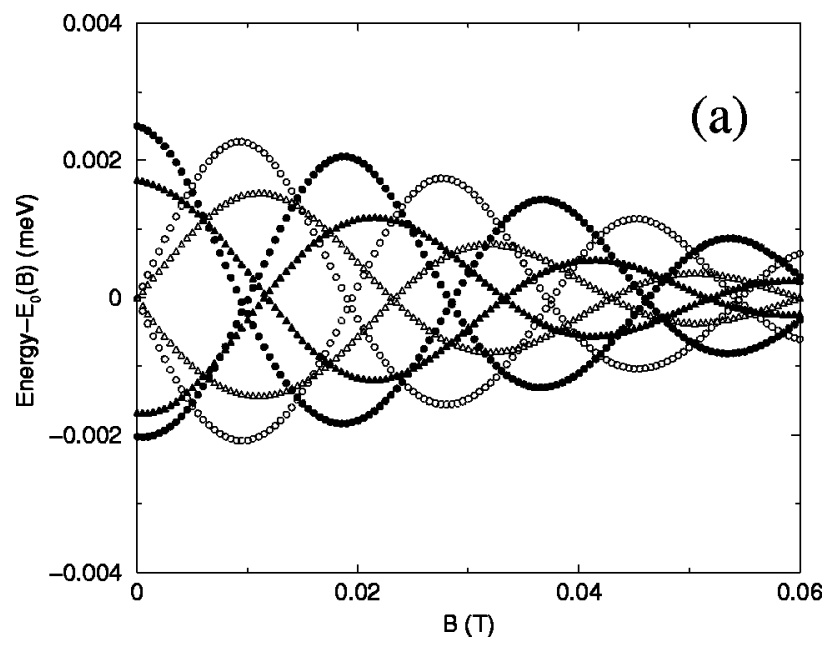

model in zero field. ${ }^{12}$ This is due to the fact that at this value of flux the Aharonov-Bohm phase acquired by the electrons when they exchange positions compensates for the sign arising from their fermionic statistics, and hence the magnetic field can be viewed as converting the electrons into "composite bosons".

A consequence of the oscillations in energy of the singlet and triplet states in the lowest multiplet is that the parity of the ground state periodically changes from symmetric to antisymmetric. Similar parity oscillations were observed by Wagner et al. and Peeters and Schweigert ${ }^{2}$ in the spectrum of two-electron quantum dots with rotationally symmetric potentials and also by $\mathrm{Ugajin}^{10}$ in a numerical study of square dots of small size. As the ground state does not flip parity in the noninteracting case, this behavior is clearly a consequence of the Coulomb interaction.

\section{CONSTRUCTION OF EFFECTIVE CHARGE-SPIN MODELS USING A HARTREE BASIS}

Although the Peierls substitution accounts for the oscillatory behavior of the lowest-energy levels, the precise details of their behavior such as the decay in amplitude of the oscillations, the marginal increase in their period (apparent from a close examination of Fig. 4), and the overall increase of the energy levels with the field, require additional explanation. This may be achieved by deriving suitable localized basis functions, and explicitly calculating the energy parameters of the resulting tight-binding (extended Hubbard) model. We have shown that this gives high accuracy for a basis set constructed from Hartree functions in the following way. We first fix the position of one electron in one corner of the square. The one-electron Schrödinger equation is then solved for the other electron, giving a set of one-electron states. For the Wigner regime considered here, the low-lying states, and in particular the ground-state, are localized near the corner of the square diagonally opposite the fixed electron. We then solve the Schrödinger equation for the first electron under the influence of the probability charge density of the other, in the sense of the Hartree method. This procedure is iterated to convergence, yielding two sets of one-

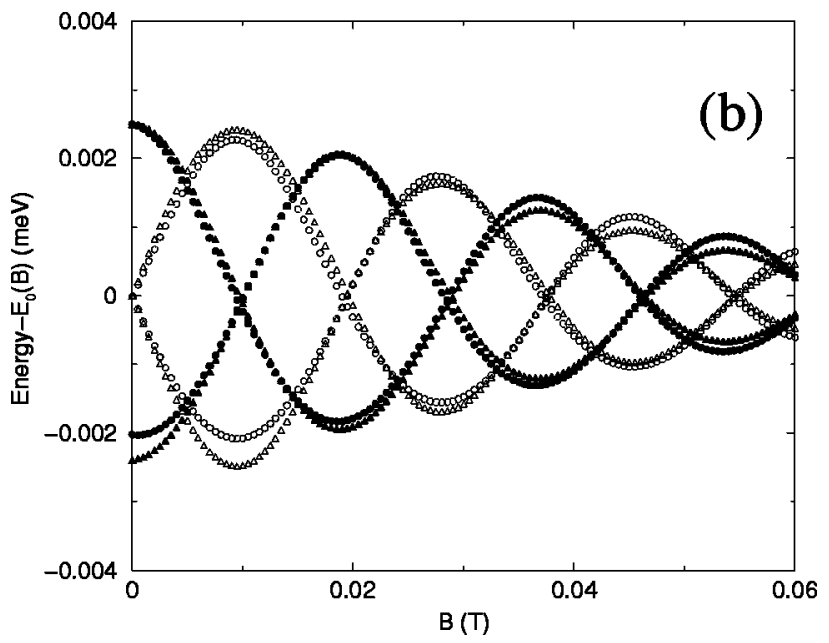

FIG. 6. Nearest-neighbor $t$ - $V$ Hubbard model (triangles) with (a) kinetic hopping only and (b) Coulomb-induced effective hopping included. Exact results (circles) are shown for comparison. Open and filled symbols indicate singlet and triplet levels, respectively. The overall increase in energy with field $\left[E_{0}(B)\right]$ has been removed. 
electron wave functions that may be mapped into one another by a rotation of $\pi$. Clearly two further sets may be deduced for the remaining corners of the square by a rotation of $\pi / 2$. Each of these four sets is composed of mutually orthogonal single-electron wave functions, but wave functions from different sets are not necessarily orthogonal to each other. A complete orthonormal set may be constructed progressively by first mutually orthogonalizing the ground state from each set using Löwdin's ${ }^{17}$ method. These states alone enable the extended Hubbard model to be constructed and, as we will show, reproduce the exact results to good accuracy. The remaining Hartree excited states may then be orthogonalized to these lowest states by the Schmidt procedure. Their effect may be accounted for later by perturbation theory, where, for the regime of interest, they give small corrections.

Restricting ourselves to the lowest four orthogonalized Hartree states we may construct all two-electron symmetric (singlet) and antisymmetric (triplet) orbital states and within this two-electron basis diagonalize the Hamiltonian (1). The results of this calculation for the lowest multiplet are shown in Fig. 5 in which we also plot the exact results for comparison. The overall increase in energy with field, which is well reproduced by the Hartree calculation, has been removed from Fig. 5. This increase in energy and the decrease in amplitude of oscillations with field may be simply related to the change in shape of the one-electron Hartree functions, which become more localized with increasing magnetic field, thereby increasing the one-electron energy while decreasing overlaps. We emphasize the importance of using Hartree rather than Hartree-Fock base states. While the latter will always give the lowest estimate of the ground-state energy for a single Slater determinant, it does not necessarily (or even usually) give a very accurate estimate of a low-lying multiplet when there is near-degeneracy. This is indeed the case for two electrons in a square dot. If we take the lowest four Hartree-Fock states (which are of course orthogonal) and form all two-electron states from these and diagonalize the Hamiltonian within this set, then, apart from the ground state, the low-lying multiplet is a very poor approximation to the exact result, with level separations that can be many orders of magnitude too large. This also gives rise to spurious qualitative errors, such as lifting the degeneracy of the two triplets at $B=0$. The reasons for this are as follows. The first two Hartree-Fock states are localized on opposite corners of a diagonal and are similar to the Hartree states. However, the next two Hartree-Fock states, which are localized near the other two diagonal corners of the square, are less localized due to the increased kinetic energy needed to ensure orthogonality. This increased width of the excited states gives rise to enhanced tunneling between the low-energy two-electron states, leading to a large increase in level separation since the tunneling matrix elements are very sensitive to the width of the localized states. Furthermore, as the symmetry of the square geometry is lost, degeneracies arising from this symmetry are lifted. Conversely, the lowest one-electron Hartree states, located near each corner of the square, are sufficiently localized to account for most of the Coulomb and kinetic energy, while still maintaining the correct symmetry.

We now consider in more detail the matrix elements of the Hamiltonian within the basis set of the lowest orthogo- nalized Hartree states and determine which are essential to reproduce the exact results to good accuracy. If we retain only the largest (diagonal) Coulomb matrix elements and the one-electron matrix elements of the kinetic energy and confining potential, then the second quantized Hamiltonian has precisely the form of Eq. (5) provided we preclude double occupation of a localized orbital, which is equivalent to setting the intra-"site" Coulomb matrix element $(U)$ to infinity. The low-energy manifold within this approximation is plotted in Fig. 6(a), together with the numerical solutions of the exact Hamiltonian, obtained in Sec. II. We see that the main source of error is in the phase of the oscillations, which have a significantly reduced frequency. Since we have only neglected off-diagonal Coulomb matrix elements in this approximation, the source of the error must arise from Coulomb-induced effective hopping. There are many such terms, most of them small. One class of terms that is not small involves three sites without spin flips. The sum of all such terms gives the following contribution to the effective Hamiltonian:

$$
\begin{aligned}
H_{3 \text { site }} & =\mathcal{P} \sum_{\substack{i j k \sigma \sigma^{\prime} \\
i \neq j \neq k}}\langle i k|g| j k\rangle c_{i \sigma^{\prime}}^{\dagger} c_{k \sigma^{\prime}}^{\dagger} c_{k \sigma^{\prime}} c_{j \sigma} \mathcal{P} \\
& =\mathcal{P} \sum_{\substack{i j k \sigma \\
i \neq j \neq k}}\langle i k|g| j k\rangle c_{i \sigma}^{\dagger} c_{j \sigma} n_{k} \mathcal{P} \\
& =\mathcal{P} \sum_{\langle i j\rangle \sigma}\left[t_{i j}^{C} c_{i \sigma}^{\dagger} c_{j \sigma}+\text { H.c. }\right] \mathcal{P},
\end{aligned}
$$

where $g$ is the Coulomb term in Eq. (1). The restriction that all three sites be different follows from the constraint of no double occupation, enforced by the projection operator, $\mathcal{P}$; $t_{i j}^{C}=\langle i k|g| j k\rangle$, independent of $k$, and we have used $P \Sigma_{k} n_{k} P=1$. Thus the effect of these Coulomb terms is to simply renormalize the one-electron hopping $t_{i j}$. Plots of the low-energy manifold are again shown in Fig. 6(b) and we see that the correct Aharonov-Bohm oscillations are reproduced to good accuracy. Thus we see that the simple nearestneighbor $t-V$ Hubbard model with $U=\infty$ describes accurately the low-energy physics provided that the Coulomb contribution to the hopping is included.

Within the $t-V$ model we see that $B$-dependent terms arise in two different ways. The Peierls factor $e^{i \phi}$ comes solely from the magnetic flux enclosed by the lattice, whereas the self-energy and the renormalization of the $t-V$ parameters originate from the physical interaction of the electrons with the magnetic field. As we reduce the size of the flux tube and approach the limit of an Aharonov-Bohm flux line, we should therefore expect the latter effects to vanish but the Peierls factors to remain. To check this supposition we display in Fig. 7 the behavior of the energy levels for flux tubes of radius $L / 2, L / 4$, and $L / 16$. It can be clearly seen that as the radius of the flux tube is reduced, the overall increase of all the energies with increasing field is reduced, corresponding to the reduction in magnitude of the self-energy. The energylevel oscillations remain, as expected, and their amplitude decays less rapidly. In the limit of an Aharonov-Bohm flux line it is clear that the amplitude of the oscillations will remain constant, and that no overall increase of energies will 

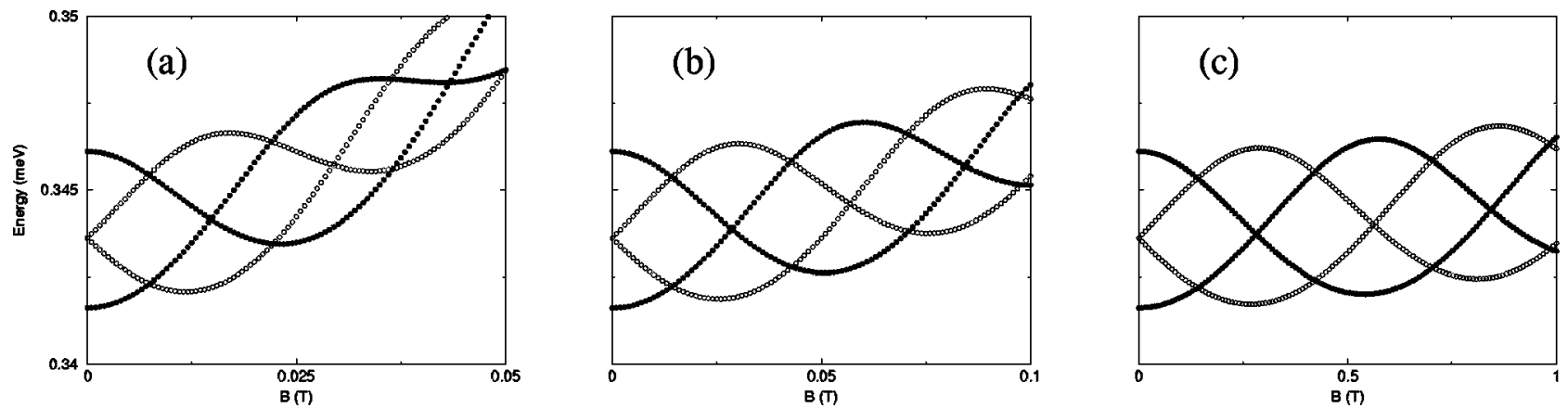

FIG. 7. Energy levels of a two-electron square-well quantum dot pierced by a flux tube. Flux-tube radii are (a) $L / 2$; (b) $L / 4 ;$ (c) $L / 16$. Filled and open circles indicate singlet and triplet levels, respectively.

occur, meaning that the system can indeed be modeled by making a pure Peierls substitution in the $t-V$ Hamiltonian.

The parallels between these results and those obtained recently by Kotlyar et al. ${ }^{15}$ for an array of coupled quantum dots holding a small number of electrons, are striking. In the quantum-dot arrays, electron localization is achieved by confining electrons to individual quantum dots, which are connected to their neighbors by leads. Exactly the same processes of localization and hopping are present in the singlequantum-dot system we consider, but localization comes from the electrostatic repulsion between the electrons, which for sufficiently large dots forces the electrons into a highly localized Wigner molecule state, and hopping occurs via tunneling processes between the various low-lying energy levels. We note, however, that the fabrication of a single large quantum dot is considerably simpler than the process of linking individual dots with leads, and the creation of a dot with the dimensions we discuss is well within current experimental capabilities. A single dot may thus provide a more convenient physical realization for studying strongly correlated mesoscopic systems than an array of dots. In addition, within a single nanostructure it should be possible to maintain coherence of the electrons for longer time scales, which is of relevance to the potential of these devices as elements of quantum computers.

\section{SUMMARY AND CONCLUSIONS}

We have studied the behavior of the low-lying energy levels of a square-well quantum dot containing two electrons, subject to a perpendicular magnetic field. It has been shown in an earlier work that an effective lattice model (a $t-V$ model) can be used successfully to treat the case of zero magnetic field, and we find that by making a simple Peierls substitution this model also predicts the main qualitative changes to the energy spectrum. This substitution gives exact results in the limit of an Aharonov-Bohm flux line, but to obtain quantitatively accurate results for other flux distributions the parameters of the model must be magnetic field dependent, resulting in an approximately quadratic increase in average energy with $B$ and a decrease in the amplitude of Aharonov-Bohm oscillations. We have justified this behavior by employing a single-electron Hartree basis in which the electrons are located close to their electrostatic minima, near diagonally opposite corners of the square. Within this framework, the overall increase in energy with magnetic field is mainly due to the increase in the one-electron Hartree energy while the reduction in amplitude of the Aharonov-Bohm oscillations is due to the increased sharpness of the localized states, which reduces the single-electron hopping. The Aharonov-Bohm oscillations are themselves a consequence of the phase change when moving from one localized state to another around the square, resulting in Peierls phase factors in the hopping matrix elements and persistent currents around the perimeter of the square. The system is thus equivalent to a tight-binding ring with four sites, the quasione-dimensionality being a consequence of Coulomb repulsion. Coulomb repulsion also has the effect of forcing the electrons to be diagonally opposite each other, causing them to rotate as a rigid pair, with an Aharonov-Bohm oscillation of twice the frequency of noninteracting electrons on a ring. The mapping to an effective lattice model is thus a valuable way of interpreting the phenomena revealed in the spectrum of the dot as the magnetic field is applied and provides, for example, an appealing interpretation of the origin of the parity oscillations of the ground state. This investigation has concentrated on the regime of weak to medium strength magnetic fields, in which the magnetic length scale is comparable with the Coulomb interaction length. Accordingly the spectrum shows a rich structure arising from the interplay between magnetic effects and correlations produced by the Coulomb interaction. As the magnetic field is increased further magnetic effects will become dominant and the eigenstates of the system will evolve toward Landau-level states. Extending the range of magnetic field to study this transition and its relation to the quantum Hall effect is an exciting prospect for future investigation.

\section{ACKNOWLEDGMENTS}

The authors would like to thank Wolfgang Häusler and Colin Lambert for stimulating discussions. C.E.C. acknowledges support from the Leverhulme Foundation and from the EV within the TMR programme. Support from the U.K. Ministry of Defense and the E.U. TMR program is also acknowledged. 
${ }^{1}$ Daniel Loss and Eugene V. Sukhorukov, Phys. Rev. Lett. 84, 1035 (2000).

${ }^{2}$ M. Wagner, U. Merkt, and A. V. Chaplik, Phys. Rev. B 45, 1951 (1992); F. M. Peeters and V. A. Schweigert, ibid. 53, 1468 (1996).

${ }^{3}$ P. A. Maksym and Tapash Chakraborty, Phys. Rev. Lett. 65, 108 (1990); P. A. Maksym and Tapash Chakraborty, Phys. Rev. B 45, 1947 (1992).

${ }^{4}$ F. Bolton, Phys. Rev. B 54, 4780 (1996).

${ }^{5}$ R. Egger, W. Hausler, C. H. Mak, and H. Grabert, Phys. Rev. Lett. 82, 3320 (1999).

${ }^{6}$ Daniela Pfannkuche, Vidar Gudmundsson, and Peter A. Maksym, Phys. Rev. B 47, 2244 (1993).

${ }^{7}$ V. Fock, Z. Phys. 47, 446 (1928).

${ }^{8}$ C. G. Darwin, Proc. Cambridge Philos. Soc. 27, 86 (1930).
${ }^{9}$ Ingibjörg Magnúsdóttir and Vidar Gudmundsson, Phys. Rev. B 60, 16591 (1999).

${ }^{10}$ Ryuichi Ugajin, Phys. Rev. B 53, 6963 (1996).

${ }^{11}$ E. P. Wigner, Phys. Rev. 46, 1002 (1934).

${ }^{12}$ J. H. Jefferson and Wolfgang Häusler, Phys. Rev. B 54, 4936 (1996).

${ }^{13}$ C. E. Creffield, Wolfgang Häusler, J. H. Jefferson, and Sarben Sarkar, Phys. Rev. B 59, 10719 (1999).

${ }^{14}$ R. E. Peierls, Z. Phys. B: Condens. Matter 80, 763 (1933).

${ }^{15}$ R. Kotlyar, C. A. Stafford, and S. Das Sarma, Phys. Rev. B 58, 3989 (1998).

${ }^{16}$ T. Demel, D. Heitmann, P. Grambow, and K. Ploog, Phys. Rev. Lett. 64, 788 (1990).

${ }^{17}$ P. O. Löwdin, J. Chem. Phys. 18, 365 (1950). 